\title{
Men's health and communities of practice in Australia
}

\begin{abstract}
Purpose: Examines the social opportunities for Aboriginal and Torres Strait Islander Men created through Men's Groups/Sheds across urban, regional and remote areas of Australia. Men's Sheds are a safe space, resembling a work-shop setting or back-yard shed, where men are encouraged to socialise and participate in health promotion, informal learning and engage in meaningful tasks both individually and at the community level.
\end{abstract}

Design/methodology/approach: Explore five case study sites through Wenger's (1998) active Communities of Practice (CoP). Qualitative methods are presented and analysed; methods comprise semi-structured interviews and yarning circles (focus groups). Five Indigenous Leaders/Coordinators participated in semi-structured interviews, as well as five yarning circles with a total of sixty-one Indigenous men.

Findings: In a societal context in which Indigenous men in Australia experience a number of social and health issues, impeding their quality of life and future opportunities, the central finding of the paper is that the effective development of social relations and socially designed programs through Men's Groups, operating as CoP, may contribute to overcoming many social and health well-being concerns.

Originality/value: Contributions will provide a better understanding of how Indigenous Men are engaging with Men’s Sheds, and through those interactions, are learning new skills and contributing to social change.

Keywords: Men’s Health, Men’s Sheds, Indigenous, Communities of Practice, Learning,

Word Count: 7805 


\section{Introduction}

Indigenous men are attending Men’s Groups/Sheds (hereinafter referred to as Men’s Sheds) in increasing numbers across urban, regional and remote areas of Australia (Cavanagh et al., 2013). The purpose of this study is to explore sustainable ways Men's Sheds can address the societal factors having a negative impact on the lives of Indigenous men with those factors contributing to current outcomes including addictions and abuse (Gracey and King, 2009, Burgess et al., 2005, Hunter, 2007). The aim of the paper is to illuminate the critical nature of the Men's Sheds in meeting the social needs of Indigenous Men. The interest in men's social well-being has grown markedly within Australia over the past decade, as scholars attempt to understand the health and well-being status of all Australian men (Smith, 2007). While the overall life expectancy and health of Australian men has improved over recent decades, the Australian Institute of Health and Welfare (AIHW, 2011) suggests men's average life expectancy remains substantially lower than that of women. Within this contest, the markedly poorer health outcomes of Aboriginal and Torres Strait Islander men are increasingly an issue of growing concern, at a societal, policy and individual level (Gracey and King, 2009, Murray and Wronski, 2006). Hunter and Jordan (2010) argue that the very concept of social inclusion raises the question of what they [meaning 'anyone', in this case Indigenous men] should be included in. Our study will argue that Men's Sheds provide a context and one opportunity to address social inclusion and issues of social connectedness, mental health and suicide prevention, to achieve better social and emotional health outcomes, which in turn may have positive impacts on the overall health and well-being outcomes of Aboriginal and Torres Strait Islander men.

This study explores the role of Men's Sheds and the impact that they have on the social well-being and health of Indigenous Men of all ages; and secondly, offers practical strategies, through social interventions within Communities of Practice (CoP) (Wenger, 1998), to enhance men's participation in the Sheds. These contributions will provide a better understanding of how Indigenous Men are engaging with Men's Sheds, and through those interactions, are learning new skills and contributing to social change. Brown (2008) challenges the notion that communities of practice and group learning has little consequence in the case of traditional learning organisations, such as schooling. Brown postulates that being a participative member learning new skills is not always constrained to a traditional teaching space such as a workplace (Jubas et al., 2016). Morgan and Nelligan (2015) explored communities of practice and through the narrative accounts of young working men argue that developing skills and creativity can happen in unique spaces such as community 
sheds and garages. Vickerstaff and Cox (2005) contend that older individuals post-work may feel disempowered but there are opportunities to develop networks and create their own experiences. Probyn (2004) encourages workplaces and community organisations to find innovative ways that garner interest and better understand Indigenous people to make positive changes to their lives. Following from the insights of this research, the current study is guided by the following research question: How do community based Men's Sheds improve the social experiences of Indigenous men and enhance their overall social and health well-being?

The organisation of this paper includes details of the background of the Men's Sheds movement, issues for Indigenous Men and their health and social wellbeing. Self-in-relation theoretical perspectives, drawing on Wenger's (1998) theoretical framework of CoP, will be introduced. This will be followed by the methodology highlighting the research question and guiding questions, data collection and analysis methods. Finally, there will be a discussion and concluding comments. 


\section{Background on the Men's Shed movement}

Men's Sheds have long been a part of Australian culture and society. With over 1,000 Men's Sheds across Australia and in excess of 90,000 members (AMSA, 2013), this is one of the largest men's community initiatives in the country (Cavanagh et al., 2013). Men's Sheds typically resemble a backyard shed or work-shop type setting, located in various places such as community halls, disused furniture factories, and garages providing the opportunity for regular hands-on activities or community programs and initiatives (Golding et al., 2007). Men's Sheds include a place specifically for Indigenous men which can house a range of facilities and services including men’s clinical services, places for discussion, 'cooling off' places, group meetings, and recreation, training and work activities (Arney and Westby, 2012) such as cultural arts and crafts that can be sold. Men's Sheds are funded through a mix of private, local council, Federal Government, and self-funded entrepreneurial ventures. Cavanagh, Bartram and McNeil (2013) describe Men’s Sheds as a central location providing mostly retired men with the opportunity to socialise and participate in meaningful tasks, such as woodwork and repairing bikes, at the individual and at the community levels. Men's Sheds also strive to provide a space where health information and services can be easily obtained. There are no two Sheds that operate the same, with some charging nominal annual fees and others with no participation fees. Furthermore, the management and facilitators within Men's Sheds play a pivotal role in the health outcomes and effectiveness of the programs (Burgess et al., 2005). Men's Sheds facilitate health interventions, 'directly through organised health checks, the distribution of information and indirectly through members yarning together and 'looking out' for one another' (Beyond Blue, 2013). Research suggests the delivery of the programs in terms of content and style, combined with adapting programs in keeping with Indigenous values and beliefs, are contributing factors to its impact (Hayes and Williamson, 2007, Sergeant, 2009). Vallance and Golding (2008) argue the Sheds provided an avenue for informal learning. Brown, Golding and Foley's (2008), research on 25 community Group/Sheds in Australia found that men learn more effectively where there was a lack of compulsion whereby 'doing projects and development peer mentoring and relationships' were important (p. 14).

Social exclusion is multifaceted and can have an impact on an individual's economic and social life (Collins, 2003). Instances of social exclusion can be found when there is a decline in an individual's engagement and their social and health-related issues increase. Individuals, such as Indigenous men, may even negotiate, interrogate and construe distinct meanings from social suggestion which is likely to be, in part, a product of their cultural history and sense of 
self. Men, such as Indigenous men, may even reject praise for self-doubt. The concept of a self-fulfilling prophecy, as explored by Merton (1948), accounts for how many individuals consider themselves as unjustifiably not worthy. A sense of learned helplessness then takes over, where individuals accept socially inappropriate labels and struggle to achieve their potential (Peterson et al., 1993).

Decades of health related research have indicated alarming rates of morbidity and mortality among Indigenous Australians (Gracey and King, 2009, Burgess et al., 2005, Hunter, 2007). According to the Australian Bureau of statistics (Australian Bureau of Statistics, 2011b) there are approximately 548,370 Australian residents identified as being of Aboriginal and Torres Strait Islander origin representing 2.5\% of the census count. The life expectancy of Indigenous Australians has stagnated at 20 years less than the national average, and is significantly worse than comparable Indigenous populations internationally (Stephens et al., 2006). Data from the Northern Territory, a federal Australian territory with the capital city of Darwin, indicates that the Indigenous burden of disease is around 2.5 times that of non-Indigenous Territorians, the 35-54 years age group being 4.1 times higher, and 37\% of Indigenous Australian's over 15 years of age having a disability or chronic health problem (Hunter, 2007). Linked to the history of colonization and settlement in Australia, the implications of the many losses suffered by Indigenous Australians have resulted in much social unrest, and mental and spiritual sorrow. The difficulties associated with belonging and adjusting to different cultural contexts has led to mental health concerns for many Indigenous Australians, particularly adjusting to western ways of life (Brown, 2001, Tsey and Every, 2000). Brown (2001) contends high levels of stress and anxiety can be a consequence of trauma and grief, and these are inextricably linked to mental health disorders, with Brown's research showing how such trauma and grief are experienced by Indigenous Australians.

Evidence indicates that Indigenous people experience important forms of social exclusion relative to the rest of the population. Data from the Survey of Education and Work (Australian Bureau of Statistics, 2013), for example, indicates that Indigenous people in general are less likely to hold a non-school qualification compared to the non-Indigenous population. Data published by the Australian Bureau of Statistics (2011a) indicates that 55.8 per cent of Indigenous people were engaged in full-time employment in 2011 compared to 76.4 percent of non-Indigenous Australians. According to the Labour Force Survey 2011, approximately 207,600 Indigenous Australians, or 38 per cent of the Indigenous population, were engaged in the labour force. Indigenous Men are more likely to be in employment compared to females (62 per cent compared to 49 per cent). Estimates indicate that 
approximately 33,800 Indigenous people aged 15 years and over were unemployed in 2011, with the highest rates of unemployment occurring in regional areas (Australian Bureau of Statistics, 2013). Government policy initiatives have been introduced, attempt to change the overall situation that Indigenous people experience but they have been relatively unsuccessful (Gunstone 2008). However, within the context of the Men's Sheds there is the potential to play at least some role in contributing to improved social and health outcomes for Indigenous men (Ang et al., 2015).

\section{Social inclusion}

There is no one accepted definition of social inclusion, but broadly it is representative of every individual's access to rights and resources without exception (Oxoby, 2009). Moreover, it is the right of every individual to feel valued, respected and included in community life (Collins, 2003) . Notions of social inclusion derived from various participatory contexts, such as a Men's Shed, can be used to explain health and wellbeing (Wilson, 2006). For example, in Indigenous Men's Groups men report better health outcomes because they have been encouraged to improve their social, cultural, and emotional wellbeing (Southcombe et al., 2013). When individuals participate in a social setting they are more likely to generate a sense of social inclusion (Shortall, 2008). Based on the interconnectedness of identity development and relationships it is important to consider one's self-definition within a social setting (Oxoby, 2009). Men make logical decisions about matters such as social justice whereas the feminine side seeks to care and protect. The way a man builds a sense of inclusion is drawn from the interactions with others, and their selfrepresentations reflected within a community of practice. That description may then influence an individual's approach to social challenges and situations and an individual may be impeded or enhanced, depending on the role within which an individual is depicted. Turner (2001) argues that social relationships between two or more individuals (male or female) within any given context, such as a Men's Shed, influence the social development of individuals. Social forces, such as community support, tend to have an ongoing effect on the development of a sense of who an individual is, such as an Indigenous man, how they know what they know, and importantly, the depth of their experiences. From this, it could be construed that men who are knowledgeable and capable of adapting within a particular social setting, such as a Men's Sheds, can gain from the community support within such a social setting. 
The words and actions of powerful others shape their individual thoughts, actions and reactions (Oxoby, 2009) and Indigenous men are particularly impacted by these environments (Cavanagh \& Bartram 2013). When the voice of powerful others, such as the leaders of Men's Sheds, is positive then individuals, such as Indigenous men, are likely to have a strong sense of self and develop successful social relationships (Southcombe, et al, 2013). In these circumstances men's experiences are shaped by the guidance they receive from their interactions with others, and their broader social experiences. We argue that it is time to start thinking and defining social inclusion more from the perspective of how individuals are afforded pragmatic experiences and the ways in which engaging in social activities can possibly make a difference to their social and health wellbeing.

\section{Communities of Practice}

Men's Sheds promote social inclusion and to explore men's social relationships with these environments we apply a theoretical framework based on Wenger's (1998) Communities of Practice (CoP) as an intervention to provide practical strategies to enhance the effective management of socially driven programs in the Men's Sheds. We argue that the key components of $\mathrm{CoP}$, mutual engagement, joint enterprise and shared repertoire, comprise a series of social processes in which social relations are a critical component. A CoP exists when people engage in a process of collective learning in a shared domain of human endeavour, learning how to do things more effectively as they interact on a regular basis (Wenger, 2007). Underpinning this perspective is the insight that learning is a situated social process that occurs through shared practices and interactions. In this regard, a CoP is a specific context within which the interactions between social relations and a sense of self as discussed in the previous section may emerge in particular ways.

Mutual engagement is the pattern and level of interaction among members of a community (Wenger, 2007). The need to provide socially supportive environments for men in the Australian community is an area of growing awareness. Scholars have identified Men's Sheds as an initiative for delivering better social and emotional health outcomes for men and in some cases boys (Golding et al., 2007). According to Wenger's (1998) model Community of Practice, Men's Sheds can function through mutual engagement, as members of the Shed establish their level of interaction. In the context of this paper, this refers more specifically to Indigenous men and health workers/educators.

Ormsby, Stanley and Jaworski’s (2010) study of older men in community-based programs found participation in Men’s Sheds positively influenced the health and well-being of men. Mutual engagement allows for increased interactions and memberships within 
organisations such as the Sheds that helps shape the group's culture and its practices (Jubas, Taber \& Brown, 2016). Smith (2007) suggests membership is more than being declared a member or being born with a characteristic; interaction is an important and imperative factor effecting mutual engagement. Hayes and Williamson (2007) suggest social benefits, such as talking about their individual issues, within the Sheds can be promoted through participation in programs that help decrease isolation and enhance self-esteem.

Joint enterprise can be applied to Men's Sheds as the common purpose that binds Indigenous Men together, as they work towards the unifying goal of overcoming negative health conditions that hold many of these men back from leading a full and healthy life. For Indigenous Men, the learning process that occurs in the Men's Shed happens through the provision of a culturally safe space to develop social skills, engage with other learning opportunities and re-connect with their Aboriginal traditions and culture. In particular, Jubas et al. (2016) promotes culture, such as Indigenous culture, as a recognised pedagogy. Individuals learn and benefit from shared experiences (Collin, 2004, Collin, 2002) and this is demonstrated in the Men's Sheds where men have the opportunities to share their stories. When the men learn new skills and gain qualifications they are better able to secure employment through employment opportunities within their communities. Because Indigenous Men are bound by a joint enterprise of cultural understandings about their issues, interventions relative to their health conditions could be adopted in the Sheds. The management and facilitators within Men's Sheds play a pivotal role in the effectiveness of programs and their health outcomes, hence strategies to create effectiveness amongst facilitators and management needs to be understood.

Shared repertoire relates to collective values, ideas and procedures of the community that bind people together and help facilitate relationships and trust. Kral and Falk's (2004) study of an Indigenous community and literacy practices uses Wenger's (1998) Community of Practice framework to assist in the development of a healthcare service. Findings suggest that for sustainable policies to be achieved they must be integrated into the social and cultural framework of the community, and must include community goals and aspirations.

Shared history suggests that repertoire is built up and shaped over time by Indigenous Men within Sheds. Allowing them to be a part of the shared history gives them a sense of belonging. This creates acceptance of intervention strategies as they can feel a part of the decision-making process and structure of the programs. As a result the likelihood of creating a desire to change is enhanced, and assists with sustainability of programs and strategies put 
in place (Gunstone 2008). The richness of shared repertoire provides a language for communicating meaning. This is particularly evident within Men's Sheds as facilitators, Indigenous and non-Indigenous learn to deliver programs and speak in a manner that is conducive to Indigenous culture and in simple language. This is important both in terms of language and terminology, to ensure cultural offences are avoided and programs can be embraced (Russ, 2008).

The overall framework for this paper combines important insights from social psychology with more recent literature on CoP to argue that an individual's sense of self is influenced by their societal context and the relationships they have developed. In turn, CoP provide a space in which that sense of self can be reimagined and reconsidered, through the creation of a space in which new relations are encountered and developed. It is these insights that will be explored in the remainder of this paper through a presentation and analysis of relevant data.

\section{Methodology}

The study employs a qualitative approach to address the meanings that individuals and groups assign to a social or human problem through an interpretive, naturalistic approach to the world (Creswell, 2007). That is, to capture context, personal interpretation and experience through understanding different realities. The study uses data derived from a study of five Indigenous Sheds conducted by a team of researchers. Semi-structured interviews were conducted with Indigenous leaders and Shed co-ordinators and five yarning circles (focus groups) with a total of 61 men. A qualitative approach was used to seek the perspectives of Indigenous Men on the community Sheds, to further develop the researchers' own understanding of the effectiveness of strategies used, gauge different perspectives and understand areas that require further attention. This was achieved through the triangulation of data using interviews with Indigenous Leaders, yarning circles with Indigenous Men and observations.

\section{Investigating Indigenous Men's Groups/Sheds}

To protect the identity of communities and Indigenous men participants we do not identify any particular town, region or names of those involved and acknowledge that this is a limitation to the study. Data was gathered from Indigenous Men in urban, regional and remote Australia in the form of interviews, yarning circles and observations. Open-ended questions were used to allow participants to answer in their own terms, and also enabled 
exploration into areas where the researchers have limited knowledge. However, this can be a time consuming technique as answers need to be coded (Bryman, 2012). Observations took place and Creswell (2013) argues they represent a key tool for collecting data in qualitative research, aiming to observe behaviour based on the research questions and purpose. Yarning circles, an Indigenous term, similar to focus groups are explicitly used for group interactions as part of the data collection method (Courtois and Turtle, 2008). This study carried out five yarning circles across five Men's Sheds. Yarning circles encourage participants to talk to one another and comment on one another's experiences and points of view (Kitzinger, 1995). Men as participants are given the opportunity to voice issues of importance to them, using a vocabulary they can understand and feel comfortable with (Rowley et al., 2000).

The research sites were selected with the guidance of an Indigenous representative and attempted to gain a cross-section of views from urban, regional and remote areas. Access to the sites was organised by the Indigenous representative, who was culturally aware and sensitive to the needs of the participants, and he accompanied the researchers and assisted in the interview and yarning circle procedures. In this regard, the researchers were cognisant of the history of 'exploitative and harmful research practices', in the context of non-Indigenous researchers ‘working with Indigenous peoples and communities’ (Guillemin et al., 2016). The researchers sought to work within the National Health and Medical Research Council (NHMRC) Values and ethics: Guidelines for ethical conduct in Aboriginal and Torres Strait Islander health research (Canberra: NHMRC; 2003), which promotes values that include respect and equality (Guillemin et al., 2016).

\section{Approach to data analysis}

Data analysis began using transcripts collected from interviews with Indigenous Leaders, yarning circles with Indigenous Men, and observations. The qualitative data was analysed using NVivo, following the steps of thematic content analysis outlined by Weber (1985). A coding framework was generated from the literature with independent coding by two researchers until data saturation to demonstrate the reliability of the coding framework (Carmines and Zeller, 1975). The reliability and validity procedures involved searching for the convergence of different sources of information to form themes from the research data and within research materials (Creswell and Miller, 2000). Categorical aggregation was used to determine themes in the data (Creswell, 2007) relative to the current role of programs delivered in the Men's Sheds, such as health and nutrition classes that focus on foods to protect against chronic diseases such as type two diabetes, and develop practical strategies 
where improvements could be made through such an intervention. Wenger's (1998) notion of a community of practice offers practical strategies; mutual engagement and interactions of men when they are involved in social programs, the joint enterprise of the men who want to achieve goals and the mutual trust through shared repertoire. Responses were further narrowed into specific themes within categories to create more concise and issue-relevant data. As Indigenous men are a vulnerable group of participants, particularly at risk of exploitation, the research team applied for ethical clearance in accordance with policy and procedure. Ethical clearance was approved by the University Human Ethics Committee.

\section{Findings: Themes in the Data}

The study discovered three main themes in regards to the role of Men's Sheds and the impact they have on the social well-being and health of Indigenous Men. The first theme relates to the 'Need for Shared Social Spaces'. The second theme relates to the 'Sharing Stories of Trauma, Grief, Incarceration'. The third theme relates to the 'Social Programs in the Sheds'.

\section{The Need for Shared Social Spaces}

Indigenous Leaders and men in yarning circles told us about the ways the Sheds have provided men with the opportunity to get in touch with their culture and have also provided a space where they can share their stories. Once Men's Sheds were established men quickly learned of their existence through what one of the leaders described as 'bush telegraph'. One of the leaders in an urban area told us the men 'need yarning circles' because as one of the participants commented he had 'no family.....no culture....need support'. Another participant told us he used to sit around watching TV....now in shed talkin to the kids'. An elderly participant announced 'the men's shed has saved me' and each of the men in this yarning circle nodded in agreement.

Moreover, Men's Sheds provide Indigenous Men with a place to go, providing them with the opportunity to focus on the future rather than consuming their energy and concerns in finding an alternative place to seek refuge. Yarning circles highlighted the restrictions that Apprehended Violence Orders (AVO) present in terms of gaining access to their children. An AVO is an order made by a court against a person who makes another person fear for their safety, to seek to protect them from further violence. Responses from men in a remote shed expressed the difficulty in this area when an AVO was forced upon them 'when you a man you don't have a home unless the woman say so' and 'it's hard to get somewhere to live'. 
Reference to the struggle experienced when attempting to find accommodation was regularly mentioned when the men told us 'they don't have anywhere to go'. Responses representative of the majority of participants suggest this is something impacting Indigenous Men since 'for us fellas it’s sharing stories', 'they need yarning circles...they've got no support', because for many Indigenous men 'it's just about surviving every day'. These concerns and stressors resulting from accommodation issues support the importance of Men's Sheds as a safe environment for Indigenous Men to seek refuge. Accommodation concerns impact on health, as Indigenous Men experience increased stress and related mental and physical health issues as they worry about a safe place to sleep. While not a complete solution in themselves, the Sheds provide at least a space for the men which otherwise would not be available

\section{Sharing Stories of Trauma, Grief, Incarceration}

Yarning circles demonstrated that high levels of trauma and grief are prevalent amongst the Indigenous Men who participated in the research, and contribute to mental health concerns. A leader of a rural shed explained 'grief is one of the biggest issues' for many men and much of the grief is related to legal matters and the stolen generation. A common response in a yarning circle was 'we all got the same fears' and 'we share deep issues' and as a result 'men suffer with depression'. For these participants, it also became evident that part of the significance of the sheds was that they provided a space in which men could discuss and work through these issues in a group or community context.

Often the social issues that men confront in their everyday lives including discrimination, racism, unemployment and violence can contribute to individual actions that result in AVOs and incarceration. Incarceration has been identified as an interactive process whereby some men with mental health issues end up in prison and being in prison attributes to ongoing mental health issues for Indigenous men (Hunter, 2007). Indigenous Men indicate that the high levels of unemployment results in increased violence and/or substance abuse as they are not afforded the opportunity to participate in a meaningful sense in society. Men participants of a yarning circle in a remote area were despondent when they were told they could not be employed, commenting that 'they [i.e. employers] say no you got a record'.

'If we got criminal record and they do criminal checks we won't get jobs'

'Wonder why we messin up, we want a go......we can prove we will be good at a job if only they would let us show them'

'I'm feelin it's not worth tryin... all I want is to forget the past' 
It was indicated by the men that AVO's often resulted in prison sentences limiting future opportunities and creating further challenges. Court orders requiring men to attend Men's Sheds instead provide a space of support and potential for engagement as expressed by leaders of two urban groups. Due to the fact the Courts have issued such orders the dynamic of men's participation has shifted from being forced to voluntarily attending.

'Don't send them to prison.....I go to the court and ask the magistrate if they can release a man to the shed'

'Bail men to the shed.....his life will be at risk in prison.....the shed is safe'

'We do circle work....gives the security and safety for men.....'

Responses further highlighted that certain prison sentences could be completed in this manner, where men can gain valuable skills through anger management and fathering programs. The men participants in the two urban groups shared their views:

'We want to lead with dignity to nurture our families.....make them proud'

'When our mob take ownership, then things will work.....prison don't own us'

'Instead of sending a brother to prison...send 'em to training program out there'

'Work in the shed to work off fines.....work gives us skills we can use'

'Men could do a program and then get a job.....this is a better alternative to going back to prison'

Responses highlight the restrictions that prison sentences impose on obtaining future education and employment opportunities, resulting in further forms of social exclusion, where social programs in Men's Sheds could work towards overcoming these issues and contribute to social inclusion. The challenges associated with AVOs and the inability to gain qualifications as a result of these convictions further highlights the need to address the social issues that the men confront through Men's Sheds.

\section{Social Programs in the Sheds}

The men's responses highlighted what they would like to learn from programs in the Sheds. This predominately involved men telling stories about their lives and focusing on some of the social determinants that impact on them. The men indicated that they want to yarn about the trauma that has happened throughout their lives and their consequent mental health and other issues issues. The men in a remote yarning circle requested:

'Teach us how to recognise when suicides might happen'

'Teach us the difference between mental health and physical health'.

'We want to know how our fellas' mental health can be improved' 
'...the biggest challenge for an aboriginal man is to communicate with other aboriginal men, to share stories...'

'Why don't we visit other groups.....and talk?'

'We need relationship services....we need safety....stolen generations...missed out...'

The motivations for other Indigenous men were to participate in programs based on receiving training where jobs could be applied for once the skills were obtained. The men in an urban shed said 'no point in training people up unless there are jobs at the end of the line' and 'if men are taught skills and don't get a job it puts them back into depression'. These kinds of comments support the need for programs that incorporate content relevant to differences amongst various Indigenous communities.

A common theme in yarning circles was the need for men to talk about their social and other issues and improve their knowledge on how to eat nutritional meals and take better care of themselves. Points were made regarding the idea that food habits they now have were acquired and embedded from a young age, making the point that 'we need nutritionists....tell us how to eat proper....how to cook' and 'we need general talks on health'.

Through interview responses from Indigenous Leaders and yarning circles with Indigenous men we heard about the underlying issues holding men back, including trauma and grief, incarceration, and identity and belonging. Yarning circles uncovered that trauma and grief can be attributed to mental health issues. Such issues were related to past experiences with ongoing impacts such as colonisation discussed in the yarning circles. Moreover, the complications that AVOs create when Indigenous men attempt to obtain a job may be a contributor to increased violence and substance abuse.

Adult literacy and education is continuously an area of concern amongst Indigenous men, with employment, income and literacy levels acting as potential factors impacting on Indigenous health. Mixed responses suggest that education is viewed as unobtainable for the improvement of health and well-being amongst men. Men members in an urban shed expressed the view that learning is for those who demonstrate high intellectual ability 'we don't have an education.....got to be smart'. Others indicated past behaviours and records prevent them from brighter futures 'education won't change anything' and 'we do it ourselves'. The men indicated there was little understanding in the broader society about Indigenous approaches, with one participant noting that we 'can't fix all communities the same way...our needs vary'. This highlights the importance of Men's Sheds to provide 
Indigenous men with the confidence to seek and embrace educational opportunities that reflect men's abilities so not to provide them with the appetite to further their knowledge.

Some of the Indigenous men from a remote location expressed thoughts about looking towards programs to fill the gap of the knowledge they have not received growing up, in the hope of gaining the skills to seek and gain employment. Interview responses with Indigenous Leaders and yarning circles with Indigenous men indicate the removal of stress and anxiety associated with housing and employment can allow for greater focus on what they need to learn.

'Teach us how to survive in the city.....get the skills.....get a job.....we need to know how to manage money' 'Men could do a program in the shed....learn new skills and then get a job....help the family....takes the stress away’

'I make boomerangs.....paint them.....it's hard to get materials.....how can we make this work?'

The researchers spoke with a Senior Aboriginal Health Education Officer and Indigenous Leader, who has been working in Indigenous Health in a remote region for many years. He reflected that, 'When the men are in a group we can get them in contact with services and it's not so much in their face'. He explained that it is sometimes difficult to get men involved because 'they think you're going to talk about gay stuff.....if you can get them there and they get started then they don't want to leave.....they sit around and tell stories and then we talk .....where men do talk about health issues'.

Indigenous Leaders across all the groups talked about the education programs that are most needed. Mental and Physical Health Educational Programs would 'provide health prevention programs and advice about how to better connect to the health services for psychological and physical needs'. Social and Emotional Programs would 'provide programs to help men build their social networks and their self-identity, sense of purpose, empowerment and self-esteem'. According to one Indigenous leader, 'We want to run programs for them [the men] in the Shed' and 'programs would need to take into consideration culture, respect and spirituality'. These comments support the need for more Men's Sheds to incorporate cultural aspects into programs they deliver and strengthen the identity and belonging of Indigenous men. The efficacy of these social environments is dependent upon Indigenous men's participation which has the potential to contribute to social and well-being outcomes. 


\section{Discussion}

This study found that strengthening social networks within Men's Sheds has a positive effect on the overall wellbeing of Indigenous men who participated in the study. The Men's Sheds can facilitate social inclusion and opportunities for social activities by providing a space in which men can come together. Hence, these environments have the potential to impact on other Indigenous men. Other opportunities can be offered by having nutritionists and healthcare workers attend the Sheds and deliver programs. Comments such as 'education....got to be smart' lends weight to the argument that whilst education is needed, many Indigenous men believe it is something out of their reach. With the attitude 'we do it ourselves' many men feel they do not have the support or opportunity to build the knowledge and skills they require for a brighter future. Moreover, past behaviours, sometimes resulting in Apprehended Violence Orders (AVOs) and prison sentences, leave men with a criminal record that results in rejection from educational opportunities, '...they say, no you got a record'. The study found that this deflates Indigenous men's confidence and appetite to make changes for the future. Some Indigenous men expressed feelings of 'I'm feelin it's not worth tryin...all I want to do is forget the past', as they are held back from their past behaviours. Golding et al. (2007) supports the ability to gain better education and increase the chance of employment through Men's Sheds, which in turn can improve overall social and wellbeing outcomes.

Yarning circles highlighted men's views on wanting and needing to work together in groups and gain knowledge in basic living skills 'When you grow up it's what you've eaten all your life.' This was further highlighted by interviews with Indigenous Leaders who suggest 'teach them how to survive in the city...don't know how to budget'. This would allow them to venture out of their communities, opening up more opportunities and options for employment. As Tsey and Every (2000) suggested, people are not born with this knowledge, and thus have to be taught skills explicitly. Tsey, Patterson, Whiteside, Baird and Barid (2002) advocate for a family well-being approach that sets out to teach skills of generic analytical and problem solving skills explicitly. The aim of this research was to engage men in ways to use their social networks and make lifestyle changes. Brown (2008, 2011) advocates for innovative ways to engage men in learning. This is something Indigenous men can achieve within Men's Sheds, by learning basic living skills (Golding et al., 2007) and extending their learning to create experiences that create long lasting life changes (Brown, 2008, 2011). 
Overall, yarning circle responses demonstrate that existing social issues and a sense of learned helplessness (Peterson et al., 1993) holds men back and that contributes to poorer health. Trauma and grief were identified by Indigenous leaders as 'one of the biggest issues'. Men made the comment 'we share deep issues' which many of the men explained has caused them to feel that they were once in a position where they were helpless, with no way of overcoming their issues. This indicates it is a common problem, and something they have gone through together. Brown (2001) agrees that high levels of stress and anxiety can be a consequence of trauma and grief, and are strongly linked to mental health disorders. Issues surrounding finding employment, accommodation, as well as trauma and grief, act as stressors that impede health and well-being (Morgan et al., 2007). There is a clear need for education programs that work towards improving the health status of Indigenous men. As Golding et al. (2007) have suggested, informal education programs need to be designed to encourage more men to participate and help build their knowledge and skills in a supportive environment. Men's Sheds promote education and community support for men and youth by offering health and social programs.

Wenger's (1998) CoP provides a framework for intervention strategies which allows men to participate in activities and interact with one another, 'For us fellas it's sharing stories'. Indigenous Leaders suggested 'they need yarning circles...they've got no support'. This is supported by Sergeant (2009) who argues interaction can provide the critical support that can be more effectively achieved through the Men's Sheds rather than if they are sent to prison. It must be noted that compulsory attendance at a Shed would be the result of a decision handed down by a Magistrate and an agreement with the Shed Co-ordinator. Yarning circles with Indigenous men revealed the thoughts of Indigenous men about the Sheds, and how law enforcers should 'bail men to the shed' as Men's Sheds could be used as a tool to mitigate some of the limitations that arise as a result of incarceration and AVO's. Similarly comments were made suggesting, 'instead of sending a brother to prison...send em to training program out there'. Indigenous Men who participated in this research believe people with AVO's resulting in prison sentences should have opportunities and support provided through the Sheds. This is supported by Golding et al. (2007) who suggest that sending men to Sheds will allow them to gain the support they require, while completing programs to ultimately seek employment. Indigenous men, who participated in this study, suggest they could 'work in the Sheds to work off fines'. Similarly, Kral and Falk's (2004) analysis would suggest that sending men to the Men's Sheds rather than prison would allow 
them to complete their conviction whilst building the knowledge and skills they require to ultimately contribute to their capacity to take control of their own lives.

\section{Mutual engagement}

Wenger's (1998) mutual engagement provides a framework for understanding the regular social interaction of men within Men's Sheds to generate shared knowledge and build valuable skills (Cavanagh et al., 2013). Ormsby et al. (2010) suggest Men’s Sheds are a safe space for men to retreat where they can interact with others and participate in knowledge sharing and building new skills. For example, in cases where men have an AVO, they are able to talk about their family problems and discuss how they can work towards overcoming legal and family issues. Through Yarning circles men expressed 'they don't have anywhere...' and 'the Men's Shed has saved me' as they are given the opportunity to learn valuable skills. Men's Sheds act as a supportive environment where men are engaged through knowledge sharing (Ballinger et al., 2009). Indigenous men share stories about difficulties, including 'hard to get somewhere to live' and 'it's just about surviving every day', whereby they can relate to one another's stressors hindering quality of life. Hayes (2001) would advocate that mutual engagement may increase the confidence and ability of Indigenous Men to focus on the social aspects of their lives, as a sense they are not alone in their hardships is created through this interaction.

\section{Joint Enterprise}

Joint Enterprise is the common purpose that connects individuals, such as men in the Sheds, as they work towards unifying goals (Wenger, 2007). Indigenous Men need to clarify their own goals, purpose and enterprise and they argue there is 'no point in training people up unless there are jobs at the end of the line.' Yarning circle responses suggest clear goals are needed to have something to work towards. Furthermore, Indigenous men believe 'you got to get training from the start'. This is supported by Morgan (2007) who contends that when training is initiated, in many cases, knowledge building happens and effective learning habits develop. An example would be a Medicare program [Australia's universal health scheme] to provide Indigenous men with knowledge and access to medical services. Through a shared domain of interest, Indigenous men can build knowledge and empower themselves to take control of their own health (Sergeant, 2009).

Communities of practice interventions may contribute to overcoming health concerns associated with substance abuse, education, literacy, incarceration as well as trauma and grief, which impact on issues of employment and mental health concerns (Morgan et al., 
2007). In order to ensure programs are well received, they need to be delivered in a learning style that responds to, and compliments, each individual. For example, Sergeant (2009) found Indigenous men are more easily engaged through observing an experienced person rather than doing it themselves. Golding et al. (2007) confirm the preferences of older men for hands-on practical learning style are more similar to those found in adult and community centres.

\section{Shared Repertoire}

Shared repertoire is the final element of Wenger's (1998) CoP and relates to the shared values, ideas and procedures of Indigenous men in the Men's Shed community. By engaging in complex tasks, such as helping to design and develop a health education intervention based on Indigenous foods, men build and enhance trust to accept, embrace and sustain educational healthcare programs (Reid and Trompf, 1992). The study found that shared repertoire assists with the overall sustainability of programs founded on men's willingness to work together (Lave and Wenger, 1991). This was highlighted in an underlying comment made by one of the Indigenous men 'can't fix all communities the same way...our needs vary'. Sergeant (2009) argues there is no 'one model fits all' approach for Indigenous communities, where generalizations can allow prejudice.

Yarning circles highlight the importance of shared history, suggesting the purpose is to be built up and shaped over time by men within Sheds (Wenger, 1998). This involves creating a sense of belonging and the importance to 'engage with men'. This is supported by Russ (2008) who found engagement and communication throughout the change process could allow clarity to be assured, as the shock of changes being made could be eased.

\section{Conclusion}

Indigenous mens' social environment and how they are described and defined has an impact on their experiences and who they are. This study explored the social opportunities afforded Aboriginal and Torres Strait Islander men through Men's Sheds. Although Sheds may be considered basic in comparison to formal facilities of learning, they represent a community meeting place where the Indigenous men who participated in this research feel comfortable to attend. In each of the five Sheds there was overwhelming support for the Men's Shed as the catalyst to enhance social opportunities and bring men together in a safe place where they pursue positive changes to their lives. Wenger's (1998) mutual engagement promotes the interaction of men within Men's Sheds to build knowledge and valuable skills (Cavanagh et al., 2013) and represents one way to provide increased opportunities for informal learning, 
social interaction and health promotion (Brown et al., 2008, Golding et al., 2007). Indigenous men hope to gain basic living skills and knowledge building and sharing that could allow them to seek employment. Indigenous Leaders discussed the concept of health, well-being and emotional programs where men can connect socially with one another strengthening their sense of identity, belonging and purpose.

Kral and Falk (2004) support the collaboration amongst Indigenous men to restore identity and social purpose. Indigenous men recognise the benefits of Men's Sheds as a place they can engage in social interaction, providing them with 'somewhere to go' and a place to build the knowledge and skills as opposed to being incarcerated. The Sheds have the capacity to meet the needs of Indigenous Men albeit in an informal way and through joint enterprise the men can work together and unify aspirations to improve their lives (Wenger, 2007). For these men, the social and informal environment helps to provide a culturally safe space to learn new skills, re-connect with their Aboriginal traditions and culture, and secure employment.

Shared repertoire can be built on community and cultural trust to encourage the men to embrace opportunities (Reid and Trompf, 1992) afforded by the Sheds. Education opportunities need to be offered to build better individual and group understandings about health and importantly to create men's desire to change (Gunstone 2008). In order for any programs to be successfully implemented, policy makers need to consider the social requests and contributions of Indigenous Men's ideas, to ensure programs reflect individual needs and are adapted accordingly. Men's Shed's programs provide one means through which Indigenous Men can develop the skills they require to seek employment, participate effectively within communities, while also gaining a sense of belonging. At the same time it is important to recognise that Men's Sheds do not operate in isolation. While they provide an important social space, as noted in the discussion above, they also need to be considered within the broader societal context of which the form a part. In a future study we will examine definitions, perceptions and challenges of Indigenous men's masculinity. We propose to conduct research through yarning circles and link data to the identity of Indigenous men. Further research could also explore in more detail the relationship between what occurs in sheds and how they relates to activities outside of the shed, to further our understanding of how CoP operate in broader societal contexts. 


\section{References}

AIHW. 2011. Ageing, disabiliy and carers [Online]. Available: http://www.aihw.gov.au/ [Accessed 8th August 2013].

AMSA. 2013. Australian Men's Shed Association [Online]. Available: http://www.mensshed.org/home/.aspx [Accessed August 1 2013].

Ang, S. H., Cavanagh, J., Southcombe, A., Bartram, T., Marjoribanks, T. \& McNeil, N. 2015. Human resource management, social connectedness and health and wellbeing of older and retired men: The role of Men's Sheds. International Journal of Human Resource Management., In press.

Arney, F. \& Westby, M. 2012. Men's places literature reveiw. Darwin, NT: Child Protection Research Program, Menzies School of Health Research.

Australian Bureau of Statistics 2011a. Australian Social Trends (No. 4102.0). Canberra: Australian Bureau of Statistics

Australian Bureau of Statistics 2011b. The Health and Welfare of Australia's Aboriginal and Torres Strait Islander Peoples (No. 4704.0). Canberra: Australian Bureau of Statistics.

Australian Bureau of Statistics 2013. Education and Work (6227.0). Canberra: Australian Bureau of Statistics.

Ballinger, M. L., Lyn, A., Talbot, L. A. \& Verrinder, G. K. 2009. More than a place to do woodwork: a case study of a community-based Men's Shed. Journal of Men's Health, 6, 120-127.

Beyond Blue 2013. Mens Sheds in Australia: Effects on physical health and mental wellbeing. Victoria.

Brown, M., Golding, B. \& Foley, A. 2008. Out the back: Mens sheds and informal learning. Fine Print, 31, 12-15.

Brown, R. 2001. Australian Indigenous mental health Australian and New Zealand Journal of Mental Health Nursing, 10, 33-41.

Brown, T. 2008. Signifying "learner", "teacher" and "mathematics": A response to a special issue. Educational Studies in Mathematics, 69, 249-263.

Brown, T. 2011. Using film in teaching and learning about changing societies. International Journal of Lifelong Education, 30, 233-247.

Bryman, A. 2012. Social Research Methods New York, Oxford University Press.

Burgess, C., Johnston, F., Bowman, D. \& Whitehead, P. 2005. Healthy country: Healthy people? exploring the health benefits of Indigenous natural resource management. Australian and New Zealand journal of public health, 29. 
Carmines, E. G. \& Zeller, R. A. 1975. Reliability and validity assessment, California, Sage Publications.

Cavanagh, J., McNeil, N. \& Bartram, T. 2013. The Australian mens shed movement: Human resource management in a voluntary organisation Asia Pacific Journal of Human Resources, 51, 292-306.

Collin, K. 2002. Development engineers' conceptions of learning at work. Studies in Continuing Education, 24, 133-152.

Collin, K. 2004. The role of experience in work and learning among design engineers. International Journal of Training and Development, 8, 111-127.

Collins, H. 2003. Discrimination, equality and social inclusion. The Modern Law Review, 66, 16-43.

Courtois, M. P. \& Turtle, E. C. 2008. Using faculty focus groups to launch a scholarly communication program. OCLC Systems and Services, 24, 160-166.

Creswell, J. 2007. Qualitative inquiry and research design: Choosing among five approaches, London, Sage.

Creswell, J. 2013. Research Design: Qualitative, Quantitative, and Mixed Methods Approaches, London, Sage Publications.

Creswell, J. W. \& Miller, D. L. 2000. Determining validity in qualitative inquiry. Theory into Practice, 39, 124-130.

Golding, B., Brown, M., Foley, A., Harvey, J. \& Gleeson, L. 2007. Mens sheds in Australia: Learning through community contexts. In: A National Vocational Education and Training Research and Evaluation Program Report (ed.). Adelaide, Australia: National Centre for Vocational Education Research (NCVER).

Gracey, M. \& King, K. 2009. Indigenous health part 1: determination and disease. 374, 6575.

Guillemin, M., Gillam, L., Barnard, E., Stewart, P., Walker, H. \& Rosenthal, D. 2016. We're checking them out: Indigenous and non-Indigenous research participants' accounts of deciding to be involved in research. International Journal for Equity in Health, 15, 114.

Gunstone , A. 2008. Australian university approaches to Indigenous policy. Australian Journal of Indigenous Education, 37, 103-108.

Hayes, R. \& Williamson, M. 2007. Men’s sheds: Exploring the evidence for best practice. Report for Office of Senior Victorians ed. Bundoora: La Trobe University.

Hunter, B. \& Jordan, K. 2010. Explaining social exclusion: Towards Social Inclusion for Indigenous Australians. Australian Journal of Social Issues, 45, 243-265. 
Hunter, E. 2007. Disadvantage and discontent: A review of issues relevant to the mental health of rural and remote Indigenous Australians. Australian Journal of Rural Health, 15, 88-93.

Jubas, K., Taber, N. \& Brown, T. 2016. Popular Culture as Pedagogy: Research in the Field of Adult Education, Rotterdam, Sense Publishers.

Kitzinger, J. 1995. Qualitative research. Introducing focus groups. British Medical Journal, 311, 299.

Kral, I. \& Folk, I. 2004. What is all the learning for? Indigenous adult literacy practices, training, community capacity and health. NCVER Commonwealth of Australia, 1, 470.

Lave, J. \& Wenger, E. 1991. Situated learning: Legitimate peripheral participation, Cambridge; Oakleigh, Vic., Cambridge University Press.

Merton, R. K. 1948. The self-fulfilling prophecy. Antioc Review, 8, 193-210.

Morgan, G. \& Nelligan, P. 2015. Labile labour-gender, flexibility and creative work. The Sociological Review, 63, 66-83.

Morgan, M., Hayes, R., Williamson, M. \& Ford, C. 2007. Men's Sheds: A community approach to promoting mental health and well-being. International Journal of Mental Health Promotion, 9, 48-52.

Murray, R. \& Wronski, I. 2006. When the tide goes out: health workforce in rural, remote and Indigenous communities. Medical Journal of Australia, 185, 37.

Ormsby, J., Stanley, M. \& Jaworski, J. 2010. Older men's participation in community-based men's sheds programmes. Health and Social Care in the Community 18, 607-613.

Oxoby, R. 2009. Understanding social inclusion, social cohesion, and social capital International Journal of Social Economics, 36, 1133-1152.

Peterson, C., Maier, S. F. \& Seligman, M. E. P. 1993. Learned helplessness: A theory for the age of personal control, New York; Melbourne, Oxford University Press.

Probyn, E. 2004. Shame in the habitus. The Sociological Review, 52, 224-248.

Reid, J. \& Trompf, P. 1992. The Health of Aboriginal Australians, Sydney, NSW, Javanovich Publishing.

Rowley, K. G., Daniel, M., Skinner, K., Skinner, M., White, G. A. \& O'Dea, K. 2000. Effectiveness of a community-directed 'healthy lifestyle' program in a remote Australian Aboriginal community. Australian and New Zealand Journal of Public Health 24, 136-144. 
Russ, T. L. 2008. Communicating change: a review and critical anlysis of programmitc and participaroty implementation approaches. Journal of Change Management, 8, 199211.

Sergeant, P. 2009. Men's Sheds-a catalyst for Indigenous men's health. The 10th National Rural Health Conference. Cairns.

Shortall, S. 2008. Are rural development programmes socially inclusive? Social inclusion, civic engagement, participation , and social capital: Exploring the differences Journal of Rural Studies, 24, 450-457.

Smith, J. A. 2007. Addressing men's health policy concerns in Australia: what can be done. Australia and New Zealand Health Policy, 4, 20.

Southcombe, A., Cavanagh, J. \& Bartram, T. 2013. Capacity Building in Indigenous Men’s Groups and Sheds across Australia. Health Promotion International, (forthcoming).

Stephens, C., Porter, J., Nettleton, C. \& Willis, R. 2006. Indigenous Health 4. Disappearing, displaces, and undervalue: A call to action for Indigeous health worldwide. The Lancet, 367.

Tsey, K. \& Every, A. 2000. Evaluating Aboriginal empowerment programs: the case of family well-being. Australian and New Zealand Journal of Public Health, 24, 509514.

Tsey, K., Patterson, D., Whiteside, M., Baird, L. \& Baird, B. 2002. Indigenous men taking their rightful place in society? A preliminary analysis of a participatory action research process with Yarrabah men's health group. Australian Journal of Rural Health, 278-284.

Turner, B. S. 2001. The erosion of citizenship. British Journal of Sociology, 52, 189-209.

Vallance, S. \& Golding, B. 2008. They're funny bloody cattle: Encouraging rural men to learn Australian Journal of Adult Learning., 48, 369-384.

Vickerstaff, S. \& Cox, J. 2005. Retirement and risk: the individualisation of retirement experiences? The Sociological Review, 53(1), 77-95., 53, 77-95.

Weber, R. P. 1985. Basic content analysis Beverly Hills CA, Sage.

Wenger, E. 1998. Communities of practice: Learning, meaning, and identity, Cambridge, UK; New York, Cambridge University Press.

Wenger, E. 2007. Communities of practice: A brief introduction [Online]. Available: http://www.ewenger.com/theory/ [Accessed 1 May 2014].

Wilson, L. 2006. Developing a model for the measurement of social inclusion and social capital in Regional Australia. Social Indicators Research, 75, 335-360. 
